\title{
Clinical Study \\ Incidental Parathyroidectomy during Total Thyroidectomy: Risk Factors and Consequences
}

\author{
Dimitrios K. Manatakis, Dimitrios Balalis, Vasiliki N. Soulou, Dimitrios P. Korkolis, \\ Georgios Plataniotis, and Emmanouil Gontikakis \\ Department of Surgical Oncology, Agios Savvas Anticancer Hospital, 11522 Athens, Greece \\ Correspondence should be addressed to Dimitrios K. Manatakis; dmanatak@yahoo.gr
}

Received 25 April 2016; Accepted 31 July 2016

Academic Editor: Giuseppe Damante

Copyright (C) 2016 Dimitrios K. Manatakis et al. This is an open access article distributed under the Creative Commons Attribution License, which permits unrestricted use, distribution, and reproduction in any medium, provided the original work is properly cited.

Objective. To evaluate the incidence of accidental parathyroidectomy in our series of total thyroidectomies, to investigate its clinical and biochemical consequences, and to identify potential risk factors. Methods. Patients who underwent total thyroidectomy between January 2006 and December 2015 were retrospectively analyzed. Pathology reports were reviewed to identify those cases who had an incidental parathyroidectomy and these were compared to patients with no parathyroidectomy, in terms of clinical (age, sex, and symptoms of hypocalcemia), pathological (thyroid specimen weight, Hashimoto thyroiditis, and malignancy), and biochemical (serum calcium and phosphate levels) factors. Results. 281 patients underwent total thyroidectomy during the study period. Incidental parathyroidectomy was noticed in $24.9 \%$ of cases, with $44.3 \%$ of parathyroid glands found in an intrathyroidal location. Evidence of postoperative biochemical hypocalcemia was noticed in $28.6 \%$ of patients with parathyroidectomy, compared with $13.3 \%$ in the no-parathyroidectomy group $(p=0.003)$. Symptomatic hypocalcemia was observed in $5.7 \%$ and $3.8 \%$, respectively $(p=0.49)$. Age, sex, thyroid specimen weight, Hashimoto thyroiditis, and malignancy did not differ significantly between the two groups. Conclusions. Our study found an association of incidental parathyroidectomy with transient postoperative biochemical hypocalcemia, but not with clinically symptomatic disease. Age, sex, thyroid gland weight, Hashimoto thyroiditis, and malignancy were not identified as risk factors.

\section{Introduction}

Standardization of the thyroidectomy technique and advances in perioperative management have led to significant decrease in the overall mortality and morbidity over the past decades. Postoperative hypocalcemia remains the single most common complication of thyroid surgery, with a prevalence ranging between 16 and 55\%, depending on the definition of low calcium levels [1-3]. Most cases are transient and asymptomatic, documented only biochemically; however permanent hypocalcemia can be debilitating for the patient, causing life-long dependency on oral calcium supplementation, significant loss of quality of life, and medicolegal issues against surgeons.

Acute parathyroid insufficiency is considered as the main factor affecting postoperative calcium levels and occurs due to incidental parathyroidectomy (IPT), parathyroid gland (PG) injury, or devascularization [3].
With an incidence of 3-30\%, IPT is a relatively common finding in thyroid pathology reports, even in the hands of experienced endocrine surgeons $[2,4]$. There is no consensus however regarding predisposing factors and high-risk patients, as well as its biochemical and clinical consequences. The aim of this study was to determine the incidence of IPT in our series, to evaluate its impact on postoperative calcium levels and to identify potential risk factors.

\section{Materials and Methods}

After institutional ethics committee approval, a retrospective analysis was undertaken, of prospectively collected data, between January 2006 and December 2015, at the Department of Surgical Oncology, Agios Savvas Anticancer Hospital, Athens, Greece. Included were all consecutive patients, aged $\geq 18$ years, with no prior history of neck exploration or irradiation, scheduled to undergo total thyroidectomy for benign or 
malignant pathology on preoperative FNA biopsy. Excluded were patients who underwent lobectomy \pm isthmectomy, subtotal thyroidectomy, cervical lymph node dissection or reoperation procedures, patients with coexisting parathyroid pathology, chronic renal impairment and other conditions affecting calcium metabolism, and patients on oral calcium supplementation.

All operations were performed by two experienced surgeons or by residents under their direct supervision, with careful dissection along the thyroid capsule, using an electrothermal bipolar vessel sealing system (LigaSure Precise or LigaSure Small Jaw, Covidien, USA) and no suture ligations. Intraoperative visualization of the recurrent laryngeal nerves and PGs was routinely attempted, but excessive dissection to search for missing PGs was avoided. After macroscopic examination, if a PG was identified within the surgical specimen, it was placed in iced saline until the end of the procedure. Following slicing into $1 \times 1 \mathrm{~mm}$ pieces, those were implanted into separate muscle pockets in the sternocleidomastoid muscles, closed by nonabsorbable Prolene $4 / 0$ sutures. The thyroidectomy specimens were wholly embedded in paraffin cubes and serial gross sections every $2 \mathrm{~mm}$ were obtained for histopathology.

Serum calcium was adjusted to preoperative albumin levels. Biochemical hypocalcemia was defined as total serum calcium concentration $<8 \mathrm{mg} / \mathrm{dL}$ (institutional reference range 8.0-10.3 mg/dL). Clinical hypocalcemia included signs and symptoms of tetany, muscle cramps, and paresthesia. Patients with permanent hypocalcemia were still on treatment more than 6 months after surgery, whereas transient hypocalcemia was defined as correction of falling serum calcium levels within 6 months postoperatively. Hyperphosphatemia was defined as serum phosphate concentration $>4.5 \mathrm{mg} / \mathrm{dL}$ (institutional reference range $2.7-4.5 \mathrm{mg} / \mathrm{dL}$ ). Serum calcium and phosphate levels were measured at 12 and 24 hours postoperatively and uncomplicated cases were discharged on the first postoperative day. Symptomatic patients and patients with evidence of biochemical hypocalcemia were initiated on an oral supplementation regime of $1000 \mathrm{mg}$ calcium carbonate/800 IU vitamin D3 three times daily and were discharged from hospital when symptoms resolved and calcium levels were restored above $8 \mathrm{mg} / \mathrm{dL}$. Hypocalcemic patients were followed up in the outpatient department at weekly intervals, with serial calcium and phosphate measurements, with gradual step-down of the supplementation dosage.

Demographic characteristics, operative findings, and biochemical test results were documented for all patients. Pathology reports were reviewed independently by two authors to determine the incidence of IPT, the number and size of PGs removed, and their location in conjunction with the thyroid capsule (extracapsular, subcapsular, and intrathyroidal), as well as final diagnosis and thyroid gland weight.

On the basis of parathyroidectomy occurrence, patients were divided into two groups (incidental parathyroidectomy, IPT group, and nonincidental parathyroidectomy, non-IPT group). Those groups were compared in terms of age, sex, thyroid gland weight, presence of thyroiditis, and malignancy in the final pathology report, as well as postoperative
TABLE 1: Demographic and pathological characteristics.

\begin{tabular}{lcc}
\hline & $n$ & $\%$ \\
\hline Sex & & \\
$\quad$ Male & 218 & $22.4 \%$ \\
Female & $54.3 \pm 14.4$ years & Range 18-85 years \\
Age & $34.9 \pm 29.1$ gr & Range 5-212 gr \\
Thyroid gland weight & & \\
Thyroid pathology & 195 & $69.4 \%$ \\
Benign & 86 & $30.6 \%$ \\
Malignant & & \\
Diagnosis & 150 & $53.4 \%$ \\
Multinodular goiter & 8 & $2.8 \%$ \\
Graves & 30 & $10.7 \%$ \\
Hashimoto & 7 & $2.5 \%$ \\
Follicular adenoma & 79 & $28.1 \%$ \\
Papillary thyroid cancer & 3 & $1.1 \%$ \\
Follicular carcinoma & 3 & $1.1 \%$ \\
Myeloid thyroid cancer & 1 & $0.3 \%$ \\
Anaplastic thyroid cancer & &
\end{tabular}

serum calcium levels and clinical, transient, and permanent hypocalcemia rates. To further assess the impact of the number of resected PGs, pre- and postoperative serum calcium and phosphate levels were compared between three subgroups (group A: no IPT, group B: one PG resected, and group C: two or more PGs resected).

Statistical analysis was performed on SPSS version 20.0, using $t$-test and ANOVA for numeric variables and chisquare for categorical variables. Statistical significance was set to $p<0.05$.

\section{Results}

In the course of the study period, a total of 281 patients (63 males, 218 females) underwent total thyroidectomy in our department (Table 1). Histopathologic examination of the resected specimen revealed benign disease and malignancy in 195 (69.4\%) and 86 (30.6\%) cases, respectively. Parathyroid tissue was verified histopathologically in 70 (24.9\%) cases (Table 2).

In most patients with IPT $(48 / 70,68.6 \%)$, only one PG was removed. However, $14(20 \%)$ and $3(4.3 \%)$ patients had two or three PGs removed, respectively. In 5 cases (7.1\%), only a fragment of parathyroid tissue was identified, after thorough examination. Mean diameter of resected PGs was $4.1 \mathrm{~mm}$

In $44.3 \%(31 / 70)$ of cases, the PGs were completely intrathyroidal, surrounded by thyroid parenchyma. In another 28 cases $(40 \%)$ they were found in a subcapsular position, while $11(15.7 \%)$ were located outside the thyroid capsule. The vast majority of extirpated PGs was normal (66/70, 94.3\%); however in 4 patients they showed mild hyperplasia without atypia. No parathyroid adenomas were discovered.

Transient biochemical hypocalcemia was evident in 48 (17.1\%) patients; however only $12(4.3 \%)$ patients presented 
TABLE 2: Incidental parathyroidectomy.

\begin{tabular}{lcc}
\hline & $n$ & $\%$ \\
\hline Incidental parathyroidectomy & 70 & $24.9 \%$ \\
Number of removed PGs & & \\
1 & 48 & $68.6 \%$ \\
2 & 14 & $20.0 \%$ \\
3 & 3 & $4.3 \%$ \\
Fragment & 5 & $7.1 \%$ \\
Autotransplantation of PGs & 4 & $5.7 \%$ \\
Mean diameter of removed PGs & $4.1 \mathrm{~mm}$ & \\
Location of removed PGs & & \\
Intrathyroidal & 31 & $44.3 \%$ \\
Subcapsular & 28 & $40.0 \%$ \\
Extracapsular & 11 & $15.7 \%$ \\
Histology of removed PGs & & \\
Normal & 66 & $94.3 \%$ \\
Hyperplasia without atypia & 4 & $0.7 \%$ \\
Adenoma & 0 & \\
\hline
\end{tabular}

TABLE 3: Comparison of incidental parathyroidectomy (IPT) and nonincidental parathyroidectomy (non-IPT) groups.

\begin{tabular}{lccc}
\hline & IPT group & Non-IPT group & $p$ value \\
\hline$N$ & 70 & 211 & \\
Age (years) & $55.4 \pm 14.7$ & $54.0 \pm 14.4$ & 0.24 \\
Sex & & & \\
$\quad$ Male & $15(21.4 \%)$ & $48(22.7 \%)$ & \\
$\quad$ Female & $55(78.6 \%)$ & $163(77.3)$ & 0.82 \\
Thyroid gland weight (gr) & $36.3 \pm 26.1$ & $34.4 \pm 30.1$ & 0.31 \\
Diagnosis & & & \\
$\quad$ Benign & $47(67.1 \%)$ & $148(70.1 \%)$ & \\
$\quad$ Malignant & $23(32.9 \%)$ & $63(29.9 \%)$ & 0.63 \\
Hashimoto thyroiditis & & & \\
$\quad$ Yes & $9(12.8 \%)$ & $33(15.6 \%)$ & \\
$\quad$ No & $61(87.2)$ & $178(84.4 \%)$ & 0.57 \\
Biochemical hypocalcemia & & & \\
$\quad$ Yes & $20(28.6 \%)$ & $28(13.3 \%)$ & \\
No & $50(71.4 \%)$ & $183(86.7 \%)$ & $\mathbf{0 . 0 0 3}$ \\
Clinical hypocalcemia & & & \\
Yes & $4(5.7 \%)$ & $8(3.8 \%)$ & \\
No & $66(94.3 \%)$ & $203(96.2 \%)$ & 0.49 \\
\hline
\end{tabular}

with clinical symptoms, which lasted up until one month postoperatively and resolved following oral calcium and vitamin D supplementation. No patient developed permanent hypocalcemia.

The univariate analysis did not identify age or gender as risk factors for IPT (Table 3). Similarly, thyroid gland weight, the diagnosis of malignancy, or the presence of Hashimoto thyroiditis were not found to correlate with IPT.
Biochemical hypocalcemia was noticed in $28.6 \%$ (20/70) of cases in IPT group and in $13.3 \%(28 / 211)$ in the non-IPT group ( $p=0.003)$. On the contrary, symptomatic hypocalcemia was observed in $5.7 \%(4 / 70)$ in the IPT group and in $3.8 \%$ $(8 / 211)$ in the non-IPT group $(p=0.49)$.

While preoperative values were similar, both calcium and phosphate levels differed significantly in the postoperative setting (Table 4 ), with significantly lower calcium ( $p=$ $0.0008)$ and higher phosphate levels $(p=0.007)$ in those patients with 2 or more PGs accidentally removed.

\section{Discussion}

Parathyroid glands were first described by Ivar Sandstrom in 1880, but it was not until 1908 that MacCallum and Voegtlin investigated their pivotal role in calcium metabolism [5].

The two upper PGs derive their origin from the fourth pharyngeal pouch and descent along with the thyroid, while the two lower PGs develop from the third pharyngeal pouch with the thymus and follow its downward migration towards the anterior mediastinum [6]. The position of the upper PGs is fairly consistent, with the cricothyroid junction and upper pole of thyroid being the most common sites. The lower PGs are more frequently ectopic and with wider distribution (lower thyroid pole, thyrothymic ligament, and upper and lower mediastinum) [7].

Supernumerary PGs are discovered in about 2-6\% of patients $[7,8]$. On the contrary, fewer than four PGs are reported in $3 \%$ of cases, but this may be partly attributed to failure of recognition of an ectopic fourth PG during dissection, rather than true agenesis of the fourth gland $[7,8]$. A large cadaveric study in a Greek population revealed 4 PGs in $93 \%$ of cases, with the vast majority of them $(>90 \%)$ being orthotopic [7]. The authors also found a notably low prevalence of intrathyroidal PGs $(0.2 \%$ of all PGs, $2 \%$ of ectopic PGs).

This variability in location, number, size, and shape renders them vulnerable to iatrogenic trauma. The incidence of IPT in our series was $24.9 \%$, which is at the upper quartile of the rates reported in the international literature (Table 5). Interestingly, we found a relatively high percentage of intrathyroidal PGs (44.3\%) compared to previously published data (Table 5). Two older studies from Greece revealed a similar prevalence of intrathyroidal PGs (42.6 and 49\%), while Sakorafas and Spiliotis reported rates of 21 and $28 \%$, respectively $[4,9-11]$.

In theory, careful examination of the thyroid gland during surgery and immediately after resection may allow for recognition of extra- and subcapsular PGs, which in turn may be autotransplanted after frozen section confirmation [6]. On the contrary, intrathyroidal PGs may lie in fissures of the thyroid surface or be truly intrathyroidal, practically precluding positive identification, even with a meticulous technique close to the thyroid capsule $[7,12]$.

However the value of systemic identification of all four PGs remains as a matter of controversy. Recent studies suggest that identifying a greater number of PGs in situ does not necessarily lead to a reduction in the postoperative rates of 
TABLE 4: Pre- and postoperative calcium $\left(\mathrm{Ca}^{++}\right)$and phosphate $(\mathrm{Ph})$ levels.

\begin{tabular}{|c|c|c|c|c|}
\hline & No PGs resected & One $P G$ resected & $\geq 2 \mathrm{PGs}$ resected & $p$ value \\
\hline Pre-op $\mathrm{Ca}^{++}$ & $9.31 \pm 0.53$ & $9.49 \pm 0.57$ & $9.37 \pm 0.56$ & 0.18 \\
\hline Post-op $\mathrm{Ca}^{++}$ & $8.65 \pm 0.71$ & $8.42 \pm 0.69$ & $7.98 \pm 0.57$ & 0.0008 \\
\hline Pre-op Ph & $3.45 \pm 0.53$ & $3.55 \pm 0.56$ & $3.55 \pm 0.60$ & 0.47 \\
\hline Post-op Ph & $3.88 \pm 0.74$ & $3.96 \pm 0.78$ & $4.52 \pm 0.84$ & 0.007 \\
\hline
\end{tabular}

TABLE 5: Previous studies on incidental parathyroidectomy (IPT).

\begin{tabular}{|c|c|c|c|c|}
\hline Name & $n$ & IPT $(\%)$ & Intrathyroidal PGs\% & Conclusion \\
\hline Özoğul et al. [13] & 801 & 2.3 & 5.2 & IPT associated with hypocalcemia \\
\hline Praženica et al. [14] & 788 & 6.6 & 20.3 & IPT associated with temporary and permanent hypocalcemia \\
\hline Qasaimeh et al. [15] & 233 & 8.6 & 21.7 & IPT associated with transient hypocalcemia \\
\hline Youssef et al. [16] & 207 & 12.6 & 57.7 & IPT not associated with hypocalcemia \\
\hline Song et al. [17] & 454 & 19.8 & - & IPT associated with transient hypocalcemia \\
\hline Erbil et al. [18] & 440 & 10.9 & 68.8 & IPT not associated with hypocalcemia \\
\hline Manouras et al. [9] & 508 & 19.7 & 49 & IPT not associated with hypocalcemia \\
\hline Sippel et al. [19] & 513 & 6.4 & - & IPT associated with biochemical hypocalcemia \\
\hline Gourgiotis et al. [10] & 315 & 21.6 & 42.6 & IPT not associated with hypocalcemia \\
\hline Sakorafas et al. [4] & 158 & 17.7 & 21 & IPT not associated with hypocalcemia \\
\hline Sasson et al. [20] & 141 & 15 & 50 & IPT not associated with hypocalcemia \\
\hline Lin et al. [21] & 220 & 9.1 & 15 & IPT not associated with hypocalcemia \\
\hline Khairy and Al-Saif [22] & 287 & 16.4 & 42.9 & IPT associated with hypocalcemia \\
\hline Sorgato et al. [23] & 882 & 7.9 & 15.7 & IPT not associated with hypocalcemia \\
\hline Abboud et al. [6] & 307 & 12 & 24 & IPT not associated with hypocalcemia \\
\hline Rix and Sinha [24] & 126 & 17.4 & 13.6 & IPT not associated with hypocalcemia \\
\hline Sheahan et al. [25] & 126 & 9.5 & - & IPT not associated with hypocalcemia \\
\hline Spiliotis et al. [11] & 315 & 10.2 & 28.1 & IPT associated with hypocalcemia \\
\hline
\end{tabular}

hypocalcemia $[12,25]$. The authors hypothesized that extensive dissection for missing PGs may cause devascularization or direct injury and may be required only in cases of central neck dissection $[17,25]$.

On the other hand, rates of permanent hypocalcemia increase when fewer than two to three PGs are preserved [26, 27]. This would justify autotransplantation of accidentally extirpated PGs $[6,28,29]$. In the present series, more than half of IPTs could theoretically have been avoided, since the PGs were found in extra- and subcapsular locations. However, when adequate parathyroid mass has been left behind, permanent hypocalcemia will rarely develop $[3,17]$.

Previous studies have variably identified younger age [14, $19,23]$, female sex $[4,9,14]$, Hashimoto thyroiditis $[18,22]$, and malignancy $[13,15,16,19,23,30]$ as potential risk factors for IPT. Of notice, Sakorafas and Gourgiotis reported lower rates of IPT in patients with malignancy, owing probably to more meticulous dissection in cases of suspected malignancy $[4,10]$. Our analysis did not reveal any of the aforementioned factors to affect the rates of IPT. In addition, more extensive procedures have been traditionally associated with higher risk for IPT, due to radicality (neck lymph node dissections) or abutment of the normal anatomical planes by scarring (reoperations) [16-24]. In this series, patients undergoing completion thyroidectomy and cervical lymphadenectomy were excluded by the study protocol.
On the other hand, we found that IPT in our patients was associated with transient biochemical hypocalcemia, but not with symptomatic disease. Evidence in the literature linking IPT to postoperative hypocalcemia and serum calcium levels remains controversial (Table 5) and may depend on the actual definitions of hypocalcemia and parathyroid failure $[1,31]$.

The number of in situ preserved PGs seems to play a role in the postoperative calcium/phosphate homeostasis. In our series, serum calcium and phosphate levels did not differ significantly between patients with none or only one PG removed. However this difference did reach statistical significance in the subgroup of patients with 2 or more PGs extirpated. Even if permanent hypocalcemia can be prevented by the preservation of a single viable PG, parathyroid autotransplantation appears as a reasonable decision in this subgroup of patients $[16,17]$.

Limitations of our study protocol include its single-institution, retrospective nature and relatively small sample size. The study population however was homogenous in terms of the surgical intervention performed, the juxtacapsular dissection technique, and the single use of hemostatic technique. On the other hand, routine postoperative PTH assessment was implemented in our department only in 2010; therefore we chose not to include it in the present analysis. Finally, the meticulous examination of the thyroid specimen and the thin 
$2 \mathrm{~mm}$ sections allowed for precise identification of parathyroid tissue, which otherwise might have been overlooked.

\section{Conclusion}

Incidental parathyroidectomy is not uncommon following thyroid surgery, even in the hands of experienced surgeons. In this 10-year audit of total thyroidectomies, incidence of IPT reached $24.9 \%$, with $44.3 \%$ of those PGs found in an intrathyroidal location, virtually precluding autotransplantation. Age, sex, thyroid specimen weight, Hashimoto thyroiditis, and malignancy were not recognized as risk factors. Incidental parathyroidectomy was associated with transient biochemical hypocalcemia, but not with clinically symptomatic disease. Patients with 2 or more PGs resected had significantly lower calcium and higher phosphate levels postoperatively, and in those cases PG autotransplantation may be beneficial.

\section{Competing Interests}

The authors declare that there are no competing interests regarding the publication of this paper.

\section{References}

[1] H. M. Mehanna, A. Jain, H. Randeva, J. Watkinson, and A. Shaha, "Postoperative hypocalcemia-the difference a definition makes," Head and Neck, vol. 32, no. 3, pp. 279-283, 2010.

[2] O. Edafe, R. Antakia, N. Laskar, L. Uttley, and S. P. Balasubramanian, "Systematic review and meta-analysis of predictors of post-thyroidectomy hypocalcaemia," British Journal of Surgery, vol. 101, no. 4, pp. 307-320, 2014.

[3] A. Sitges-Serra, S. Ruiz, M. Girvent, H. Manjón, J. P. Dueñas, and J. J. Sancho, "Outcome of protracted hypoparathyroidism after total thyroidectomy," British Journal of Surgery, vol. 97, no. 11, pp. 1687-1695, 2010.

[4] G. H. Sakorafas, V. Stafyla, C. Bramis, N. Kotsifopoulos, T. Kolettis, and G. Kassaras, "Incidental parathyroidectomy during thyroid surgery: an underappreciated complication of thyroidectomy," World Journal of Surgery, vol. 29, no. 12, pp. 1539$1543,2005$.

[5] W. G. MacCallum and C. Voegtlin, "On the relation of tetany to the parathyroid glands and to calcium metabolism," Journal of Experimental Medicine, vol. 11, no. 1, pp. 118-151, 1909.

[6] B. Abboud, G. Sleilaty, C. Braidy et al., "Careful examination of thyroid specimen intraoperatively to reduce incidence of inadvertent parathyroidectomy during thyroid surgery," Archives of Otolaryngology-Head and Neck Surgery, vol. 133, no. 11, pp. 1105-1110, 2007.

[7] D. Lappas, G. Noussios, P. Anagnostis, F. Adamidou, A. Chatzigeorgiou, and P. Skandalakis, "Location, number and morphology of parathyroid glands: results from a large anatomical series," Anatomical Science International, vol. 87, no. 3, pp. 160164, 2012.

[8] G. Akerstrom, J. Malmaeus, and R. Bergstrom, "Surgical anatomy of human parathyroid glands," Surgery, vol. 95, no. 1, pp. 14-21, 1984.
[9] A. Manouras, H. Markogiannakis, E. Lagoudianakis et al., "Unintentional parathyroidectomy during total thyroidectomy," Head and Neck, vol. 30, no. 4, pp. 497-502, 2008.

[10] S. Gourgiotis, P. Moustafellos, N. Dimopoulos, G. Papaxoinis, S. Baratsis, and E. Hadjiyannakis, "Inadvertent parathyroidectomy during thyroid surgery: the incidence of a complication of thyroidectomy," Langenbeck's Archives of Surgery, vol. 391, no. 6, pp. 557-560, 2006.

[11] J. Spiliotis, A. Vaxevanidou, F. Sergouniotis et al., "Risk factors and consequences of incidental parathyroidectomy during thyroidectomy," The American Surgeon, vol. 76, no. 4, pp. 436-441, 2010.

[12] P. Praženica, K. O’Driscoll, and R. Holý, "Incidental parathyroidectomy during thyroid surgery using capsular dissection technique," Otolaryngology_Head and Neck Surgery, vol. 150, no. 5, pp. 754-761, 2014.

[13] B. Özoğul, M. N. Akçay, A. Kisaoğlu, S. S. Atamanalp, G. Öztürk, and B. Aydinli, "Incidental parathyroidectomy during thyroid surgery: risk factors, incidence, and outcomes," Turkish Journal of Medical Sciences, vol. 44, no. 1, pp. 84-88, 2014.

[14] P. Praženica, L. O’Keeffe, and R. Holý, "Dissection and identification of parathyroid glands during thyroidectomy: association with hypocalcemia," Head and Neck, vol. 37, no. 3, pp. 393-399, 2016.

[15] G. R. Qasaimeh, S. Al Nemri, and A. K. Al Omari, "Incidental extirpation of the parathyroid glands at thyroid surgery: risk factors and post-operative hypocalcemia," European Archives of Oto-Rhino-Laryngology, vol. 268, no. 7, pp. 1047-1051, 2011.

[16] T. Youssef, G. Gaballah, E. Abd-Elaal, and E. El-Dosoky, "Assessment of risk factors of incidental parathyroidectomy during thyroid surgery: a prospective study," International Journal of Surgery, vol. 8, no. 3, pp. 207-211, 2010.

[17] C. M. Song, J. H. Jung, Y. B. Ji, H. J. Min, Y. H. Ahn, and K. Tae, "Relationship between hypoparathyroidism and the number of parathyroid glands preserved during thyroidectomy," World Journal of Surgical Oncology, vol. 12, no. 1, article 200, 2014.

[18] Y. Erbil, U. Barbaros, N. Ozbey, F. Aral, and S. Özarmağan, "Risk factors of incidental parathyroidectomy after thyroidectomy for benign thyroid disorders," International Journal of Surgery, vol. 7, no. 1, pp. 58-61, 2009.

[19] R. S. Sippel, Ö. Özgül, G. K. Hartig, E. A. Mack, and H. Chen, "Risks and consequences of incidental parathyroidectomy during thyroid resection," ANZ Journal of Surgery, vol. 77, no. 1-2, pp. 33-36, 2007.

[20] A. R. Sasson, J. F. Pingpank Jr., R. W. Wetherington, A. L. Hanlon, and J. A. Ridge, "Incidental parathyroidectomy during thyroid surgery does not cause transient symptomatic hypocalcemia," Archives of Otolaryngology Head and Neck Surgery, vol. 127, no. 3, pp. 304-308, 2001.

[21] D. T. Lin, S. G. Patel, A. R. Shaha, B. Singh, and J. P. Shah, "Incidence of inadvertent parathyroid removal during thyroidectomy," Laryngoscope, vol. 112, no. 4, pp. 608-611, 2002.

[22] G. A. Khairy and A. Al-Saif, "Incidental parathyroidectomy during thyroid resection: incidence, risk factors, and outcome," Annals of Saudi Medicine, vol. 31, no. 3, pp. 274-278, 2011.

[23] N. Sorgato, G. Pennelli, I. M. Boschin et al., "Can we avoid inadvertent parathyroidectomy during thyroid surgery?" In Vivo, vol. 23, no. 3, pp. 433-440, 2009.

[24] T. E. Rix and P. Sinha, "Inadvertent parathyroid excision during thyroid surgery," Surgeon, vol. 4, no. 6, pp. 339-342, 2006. 
[25] P. Sheahan, R. Mehanna, N. Basheeth, and M. S. Murphy, "Is systematic identification of all four parathyroid glands necessary during total thyroidectomy?: a prospective study," Laryngoscope, vol. 123, no. 9, pp. 2324-2328, 2013.

[26] F. Pattou, F. Combemale, S. Fabre et al., "Hypocalcemia following thyroid surgery: incidence and prediction of outcome," World Journal of Surgery, vol. 22, no. 7, pp. 718-724, 1998.

[27] O. Thomusch, A. Machens, C. Sekulla, J. Ukkat, M. Brauckhoff, and H. Dralle, "The impact of surgical technique on postoperative hypoparathyroidism in bilateral thyroid surgery: a multivariate analysis of 5846 consecutive patients," Surgery, vol. 133, no. 2, pp. 180-185, 2003.

[28] T. Wei, Z. Li, J. Jin et al., "Autotransplantation of Inferior Parathyroid glands during central neck dissection for papillary thyroid carcinoma: A Retrospective Cohort Study," International Journal of Surgery, vol. 12, no. 12, pp. 1286-1290, 2014.

[29] T. Kikumori, T. Imai, Y. Tanaka, M. Oiwa, T. Mase, and H. Funahashi, "Parathyroid autotransplantation with total thyroidectomy for thyroid carcinoma: long-term follow-up of grafted parathyroid function," Surgery, vol. 125, no. 5, pp. 504508, 1999.

[30] N. S. Campos, L. P. Cardoso, R. T. Tanios et al., "Risk factors for incidental parathyroidectomy during thyroidectomy," Brazilian Journal of Otorhinolaryngology, vol. 78, no. 1, pp. 57-61, 2012.

[31] L. Lorente-Poch, J. J. Sancho, J. L. Muñoz-Nova, P. SánchezVelázquez, and A. Sitges-Serra, "Defining the syndromes of parathyroid failure after total thyroidectomy," Gland Surgery, vol. 4, no. 1, pp. 82-90, 2015. 


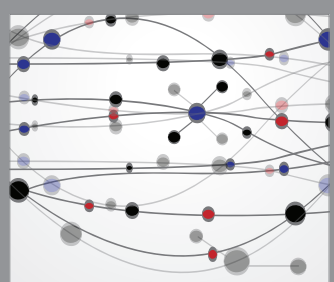

The Scientific World Journal
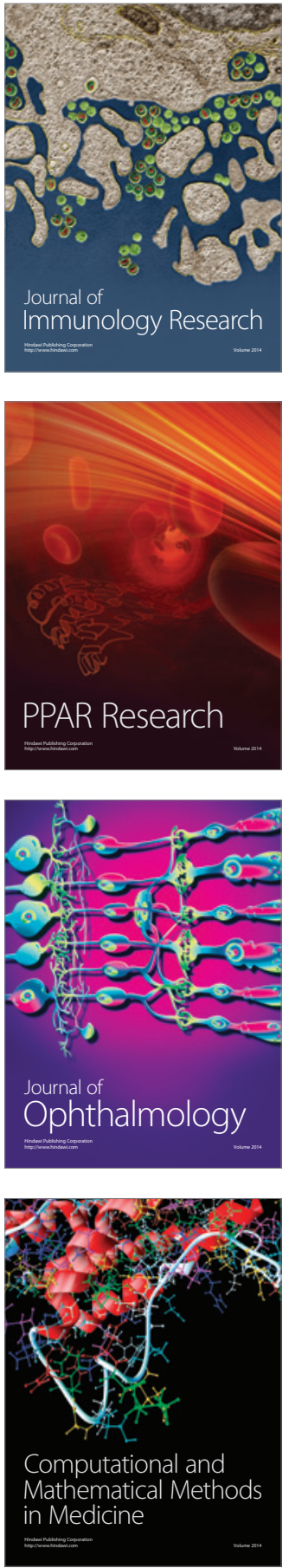

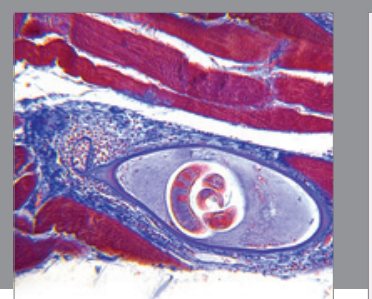

Gastroenterology Research and Practice

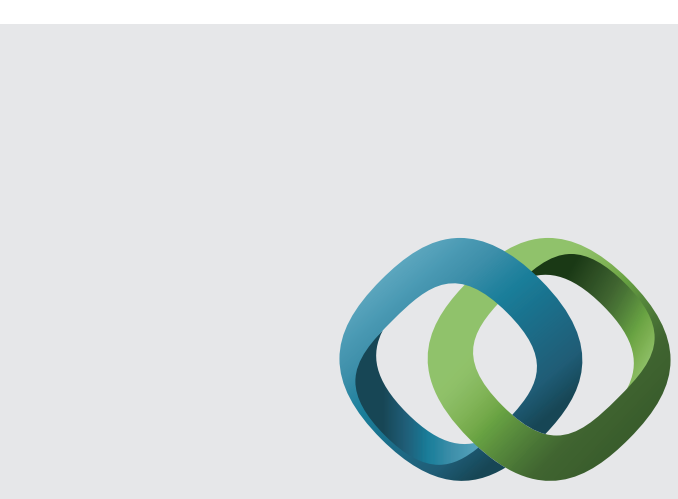

\section{Hindawi}

Submit your manuscripts at

http://www.hindawi.com
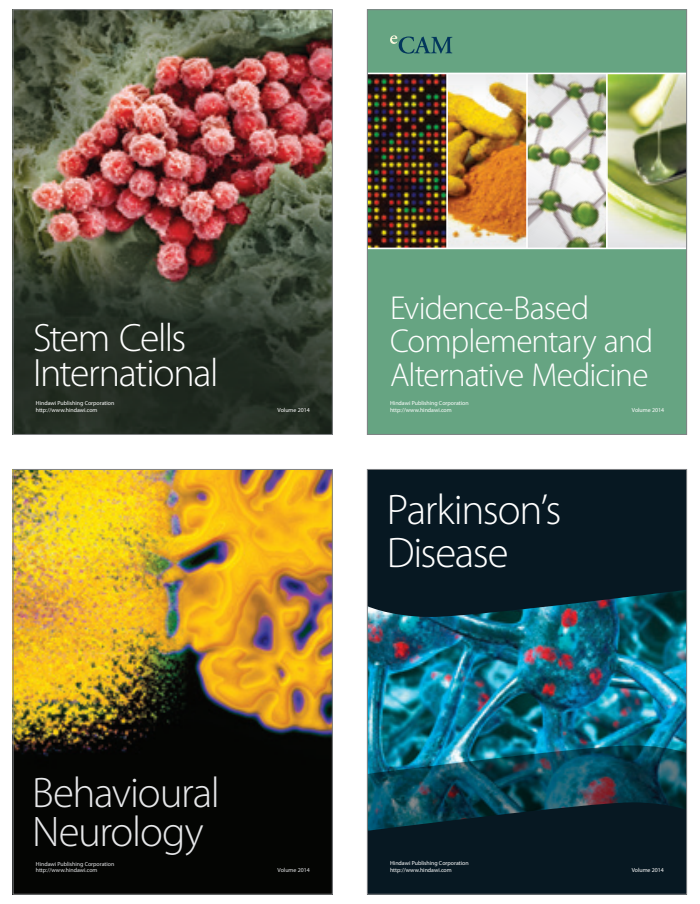
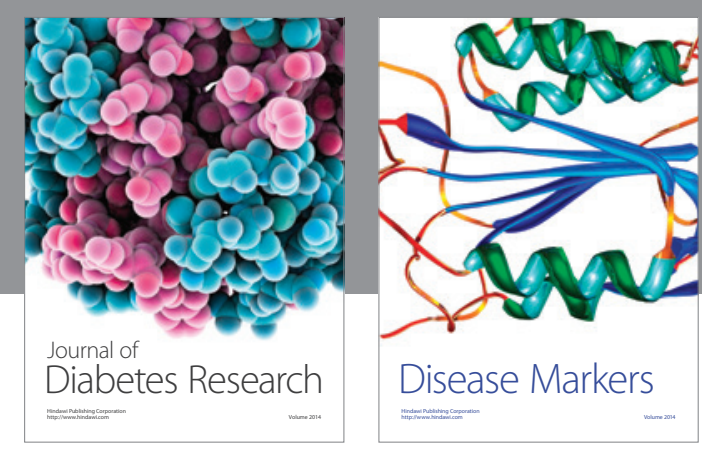

Disease Markers
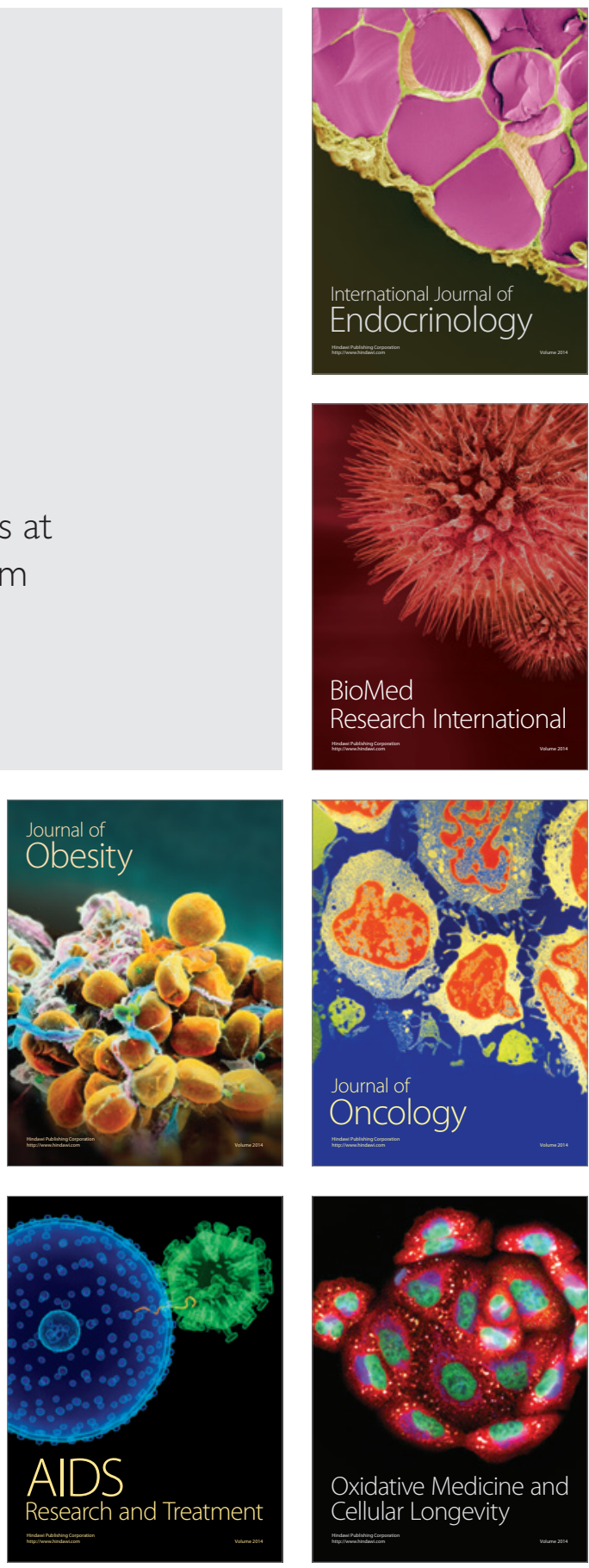